\title{
AN INVESTIGATION OF VIDEO COMMUNICATION OVER BANDWIDTH LIMITED PUBLIC SAFETY NETWORK
}

\author{
Raja Wasim Ahmad ${ }^{\text {* }}$, Rafi us Shan ${ }^{1}$, Atta ur Rehman Khan ${ }^{2}$, Kashif Bilal, \\ Junaid Shuja ${ }^{1}$,Shahzad Sarwar ${ }^{3}$,Torki A.Altameem ${ }^{4}$, Sajjad A. Madani ${ }^{1}$ \\ ${ }^{1}$ Department of Computer Science, COSMATS Institute of Information Technology, Pakistan. \\ ${ }^{2}$ Department of Computer Science, Air University, Islamabad, Pakistan \\ ${ }^{3}$ Punjab University College of Information Technology, University of Punjab \\ ${ }^{4}$ Department of Computer Science, Riyadh Community College, King Saud University \\ Email: wasimraja,shan,kashifbilal,junaidshuja,madani\}@ciit.net.pk,dr@attaurrehmankhan.com \\ s.sarwar@pucit.edu.pk,altameem@KSU.EDU.SA
}

DOI: https://doi.org/10.22452/mjcs.vol31no2.1

\begin{abstract}
Application performance of video-based services for public safety and security (PSS) systems is of high importance in order to protect citizens, organizations, and institutions against threats to their well-being. Video based services help PSS personnel to efficiently perform their rescue operations. Real-time video streaming of incident-based services over the PSS communication networks facilitates in realistic assessment of the situations and saves many precious lives. However, video communication over PSS networks, such as Terrestrial Trunked Radio (TETRA), is a big challenge due to the narrowband and the hostile environment. Thus, specialized codecs have been developed considering the constraints of the PSS networks. This paper presents performance comparisons of two video codecs, namely H.264/AVC and MPEG-4, over TEDS links. The significance of the results is evaluated using SPSS (t-test) to critically analyse the data. It is observed that video quality offered by H.264/AVC over TEDS links is better than the one offered by MPEG-4. Moreover, it is also noticed that TEDS bandwidth limitations substantially affect the quality of reconstructed video containing frequent changes of scenes. Based on the findings of our analyses, we propose a set of applications which are suitable for TEDS networks. The findings of this study would help the researchers to further explore the possibilities of video communication over TEDS links by optimizing the lower level network technologies.
\end{abstract}

Keywords: TETRA, Public Safety and Security (PSS), video, Jitter, delay

\subsection{INTRODUCTION}

Public Safety and Security (PSS) system ensures safeguard of the public from all kinds of natural and artificial cataclysms, and takes necessary defensive measures wherever possible [1]. Public safety agents including fire brigade, maritime, armed forces, or coastline guards, being the first responders to emergency situations, guarantee public safety during rescue operations. Safety agents exploit the PSS systems to co-ordinate their rescue efforts during emergency situation using telecommunication infrastructure to efficiently perform their rescue duties [2]. As an example, consider a bomb blast rescue operation wherein a centralized command keeps track of the rescue personnel locations and updates the condition of victims to the medical teams, if any survivors found, in a timely fashion. However, for mission-critical situations, a reliable communication plays crucial role as (during rescue operations) public safety, which highly relies on degree of the coordination among rescue teams. 
Public Safety and Disaster Recovery (PSDR) organizations exploit the professional mobile radio (PMR) communication systems to perform their duties more safely and efficiently. TETRA [3, 4] and Project 25 [5] (also known as P25) are two dominant and mature PMR communication systems with unique features to fulfil the requirements of PSDR agencies. PMR communication systems are used by government administration and law enforcement agencies to offer numerous vivacious services such as search and rescue, ambulance tracking, and fire-fighting [6]. The recent advancements in PMR communication system assists in monitoring a rescue operation and guiding the on-field PSS professionals from the control room via multimedia communication services. Furthermore, real-time images of the rescue operations such as fire incident, flood, bomb blast, and earthquake can be fed to the control room in order to facilitate analysis of on-ground situation. Visual illustrations are more expedient to analyse situations compared to an audio of an event [5, 7]. For instance, consider a scenario in which a bank raid is reported to police headquarters. In response, the on-field policemen can access bank cameras trough TETRA which is further connected to the bank's network. In this scenario, a few TETRA slots may be used for video transfer and the remaining slots can be used for audio and image transfer [8]. Recently, many video-based services are designed using well-known video codecs which support variable frame rate and deliver desired quality video outputs to facilitate professionals. However, delivering desired quality-of-service (QoS) [9] as demanded by PSDR services is a challenge for narrowband TETRA system [4, 5]. Moreover, the PSDR-based technologies offer enormous features such as bearer services [2, 10], tele services, fleet services, telephone interconnect, and group call support to enable the PSS agents to efficiently coordinate with each other. Among others, TETRA is a well-known PSDR standard opted by several PMR organizations to perform rescue operations efficiently. The unique features of TETRA include fast call setup, fast message transmission, priority-based call handling, advance encryption, and mutual authentication. Further, TETRA ensures reliability and high quality of services using numerous modulation schemes, mapped against application requirements such as $\frac{\pi}{4}$ DQPSK, D8PSK, 16QAM, 64QAM, etc. TETRA-II or TEDS is evolved from TETRA-I in order to facilitate high rate data applications such as medical data and multimedia-based services [6].

The well-known video communication-based applications including multimedia messaging, video-telephony, videoconferencing over mobile TV, wireless and wired Internet video streaming, standard and high definition TV broadcasting are resource-expensive in terms of their processing demands and bandwidth requirements. To cater the requirements of these applications, a variety of video transmission and storage systems with unique features are employed [7]. However, the video communication applications demand larger band width compared to audio communication. For instance, Quadrature Common Inter-Frame (QCIF) raw video demands a bandwidth of 7 Mbps. Nevertheless, to meet required QoS, the bandwidth requirement can be reduced to as low as $5 \mathrm{Kbps}$ [7] by employing state-of-the-art video compression codecs that are designed to compress application contents for bandwidth limited/narrowband systems $[6,11]$.

The paper evaluates the performance of H.264/AVC and MPEG-4 video codecs over TEDS network links. TEDS is a new TETRA High Speed Data (HSD) service incorporated in TETRA-II. To achieve higher data rates, TEDS uses high order modulation schemes (discussed in Section 2.1). H.264/AVC and MPEG-4are suitable for bandwidth-limited networks due to excellent error resilient features to recover corrupted video frames during video communication [5]. H.264/AVC [12] is block-based, whereas MPEG-4 [13] is an object-based video codec. MPEG-4 is flexible and robust in nature and supports bit-rates of as low as $5 \mathrm{Kbps}$ for mobile applications, and as high as $4 \mathrm{Mbps}$ for TV/film applications. Moreover, we have proposed a simulation framework, named TETRA Network Simulation Framework (TENS), to investigate codecs performance. Furthermore, we propose several multimedia applications that maybe conveniently executed over the TEDS.

The rest of the paper is organized as follows: section 2 provides an overview of TETRA, TEDS, and its protocol stack; section 3presents the related work; section 4 presents the proposed simulation framework, selected codecs and their qualitative comparison; section 5presents simulation setup, comparison parameters, results and recommendations; and, section 6 concludes with discussions and future research directions. 


\subsection{BACKGROUND}

The PMR standard delivers secure group call and direct mode communication [1] features to satisfy PSS agents communication needs. The Direct Mode Operation (DMO) feature enlarges PMR network coverage range by augmenting capabilities of a mobile station (MS) to act as a relay for the MS located outside network coverage range. TETRA is the outcome of the PMR standardization with unique features to support PSS agents to efficiently perform their duties during rescue operations $[6,14]$. The following section discusses the features of TETRA network with special emphasis on highlighting the variances between TETRA and TEDS. Further, TEDS protocol stack is discussed to highlight mobile to mobile communication over IP.

\subsection{Terrestrial Trunked Radio (TETRA)}

TETRA [3] is a true digital technology which have been accepted worldwide due to its differentiating features to assist rescue teams during emergency scenarios. Unlike other mobile technologies, TETRA is differentiated by a large area of practicable radio coverage via a lower frequency band $(5 \mathrm{MHz})$. It offers high quality voice communication using the digitally-coded excited linear predictive (CELP) voice codecs, and is extremely resilient to background noise [3, 15]. Furthermore, TETRA system employs dynamic control and distribution of communication resources to insure high availability during rescue operations. The system is highly flexible due to its support for trunked mode operations (TMO) and direct mode operations (DMO) [5].Currently, TETRA has two releases, namely TETRA-I and TETRA-II. TETRA-I supports voice, short data messages, and automatic vehicle location (AVL) services for monitoring and tracking vehicles in a transport system. Alternatively, TEDS (TETRA-II) has evolved from TETRA-I, and supports emerging high data rate applications, such as emergency video calls and multimedia services. The differentiating features of TEDS include adoption of multicarrier filter-based signalling, incorporation of multi-level spectrum efficient modulation schemes, powerful turbo codes, inclusion of efficient link adaptation schemes, multi-carriers $(25 \mathrm{KHz}, 50$ $\mathrm{KHz}, 100 \mathrm{KHz}$, and150 KHz spacing) support, and adaptive modulation schemes, such as the quadrature amplitude modulation (QAM-4, QAM-16, QAM-64), the differential quadrature phase shift keying ( $\frac{\pi}{4}$ DQPSK, D8PSK), and sector antennas for increasing coverage. In essence, TEDS is capable of providing enhanced data rates, coverage, and improved data security by eliminating masquerade attacks $[5,16]$. TEDS $[12,17-19]$ supports data rates up to $300 \mathrm{kbps}$ which is approximately ten time higher than TETRA-I [18, 20-22].TEDS has become an attractive system for the PSS agents and is applicable in a wide variety of areas such as telemedicine, real-time multimedia transfer, and live map tracking. However, videos over TEDS may be subject to QoS degradation due to the bandwidth limitations. Table 1 highlights the differences between TETRA 1 and TEDS networks.

Table 1: Comparison between TETRA 1 and TEDS

\begin{tabular}{|l|l|l|}
\hline Parameter & TETRA & TEDS \\
\hline Modulation & $\frac{\pi}{4}$ DQPSK & $\begin{array}{l}\left(\frac{\pi}{4} \text { DQPSK, D8PSK), QAM-4,QAM- }\right. \\
64, \text { QAM-16 }\end{array}$ \\
\hline Channels Capacity & $25 \mathrm{KHz}$ & $25,50,100,150 \mathrm{KHz}$ \\
\hline Data Rate & $36 \mathrm{kbps}$ & $300 \mathrm{kbps}$ \\
\hline Communication Mode & TMO & DMO and TMO \\
\hline Coverage & Low & High \\
\hline
\end{tabular}

\subsection{TEDS Protocol Stack}

TEDS is a spectrally-effective, integrated, and high speed data service that is scalable to wideband and ultimately broadband rates. TEDS protocol stack implements computer networking protocol suite that determines the interoperability of a layered model. As depicted in Fig. 1, switching and management infrastructure (SWMI) acts as a relaying entity between two communicating mobile terminals. 




Fig. 1: TEDS Protocol Stack

In TEDS protocol stack (see Fig. 1), IP acts as a transport medium to connect source mobile terminal to the SWMI and the destination mobile terminal. The Multimedia Exchange (MEX) layer handles the control and data traffic between MEX and the Sub-Network Dependent Convergence Protocol (SNDCP). On MEX queues, packets are processed on the basis of the priority level of different applications [3,21]. The SNDCP layer assists communicating applications to activate, deactivate, and modify the context in addition to controlling data traffic to the lower entities. The Mobile Link Entity (MLE) is responsible for presenting the sub-network services to the higher network layer entities according to the MLE service description; handling signal capability measuring; and handing over related decisions. Moreover, MLE routes the packets between a Mobile Terminal (MT) and the Terminal Equipment (TE) entity. The Air Interface (AI) delivers data and control packets to the communicating entities [3, 23, 24].

\subsection{RELATED WORK}

MPEG-4 exploits low bit rate and error resilient tools to overcome the effect of error-prone nature of TETRA channels. In [6], the authors discuss the performance of the video communication system by transferring MPEG-4 encoded video over TETRA links. It presents a detailed discussion on effect of error-resilient features of MPEG-4 on reconstructed video quality at receiver' send. The simulations were performed using TETRA TCH/4.8 and TCH/7.2 channels, 176x144 (QCIF) video frame resolution, $5 \mathrm{fps}$ frame rate, and IPPPIPPPP video frame sequence (discussed in Section 4.2) to ensure a constant throughput rate of $14 \mathrm{Kbps}$. Further, to achieve optimal performance, error-resilient features were enabled with resynchronization marker added after every 800 coded bits. In addition, multipath propagation condition (TU 50 channel) and Additive White Gaussian Noise (AWGN) were used during simulations. The study revealed that the Average Peek Signal-to-Noise Ratio (APSNR) increases significantly when more error-resilient tools are used in combination. Also, the APSNR, data partitioning (DP), and Reversible Variable Length Codes (RVLC) are 
much higher than the APSNR obtained using the DP alone. The negative point of this study is that the authors did not play the video at the receiver's end to draw a conclusion about suitability of video over TETRA.

In [7], the authors recommend to compress videos prior to transfer over TETRA links as it improves both the network and application performance by reducing the video contents. The authors highlighted the role of video codec's errorresilient features to improve video communication performance. For instance, MPEG-4 employs video Packet Resynchronization Marker (RM), Data Partitioning (DP), Reversible Variable Length Codes (RVLC), and Header Extension Code (HEC) to handle damaged video frames during video communications over TETRA channels (TCH/4.8 and $\mathrm{TCH} / 7.2$ ). In this study, same simulation settings were used as discussed in [7], in exception to the video sequence, which was changed to IPPPPPPPPPI. It indicates that among video sequence, the 11th frame is an I-frame. The inclusion of HEC-resilient tool improved APSNR due to its support for reconstruction of the damaged frames. The study highlighted that PSNR available on rate controller punctured (FEC mechanism) codes channel at receiver's end is higher than the channels without rate controller, due to the bit loss recovery of the former. Moreover, it was observed that with low frame rate, higher PSNR can be achieved on TETRA links. Despite being effective in error recovery, the error coding schemes have the tendency to reduce throughput. However, to run error resilient features on TETRA mobiles, high computational power is required to reconstruct a video in real time.

In [25], the authors discuss several error-resilient tools and protection codes for TETRA network. In TETRA, Rate Compatible Punctured Convolution (RCPC) is advised for protection from errors at the cost of reduction in net data rate from $7.2 \mathrm{Kbps}$ to $2.4 \mathrm{Kbps}$ and $4.8 \mathrm{Kbps}$ for high and low data protection schemes, respectively. INTRAFRAME is a method to handle packet loss but it reduces the compression efficiency significantly. Therefore, instead of INTRAFRAME, synchronization point method (Reed Solomon (RS)) is exploited as an alternate. However, INTRADCFRAME produces better results in terms of video quality, as compared to $1 / 3 \mathrm{RS}$ codes. It is observed that when different error codes (RS, INTRADC) are used in combination, the overall video quality improves. The authors also investigate shortened RS, RCPC, and concatenated RS/convolution codes on typical TETRA urban channels. It was noticed that the performance of convolution and RS is approximately the same in many of the scenarios except the burst error scenario, where the RS code offers better performance than others.

A non-predictive video codec improves application performance when applied to low-bandwidth networks such as TETRA. The scheme proposed in [26] omits the extra prediction steps to achieve a robust, low bit rate video communication. To do so, the video sequences were grouped into a number of frames, called Group of Frames (GOP), and the identical sub-bands from each frame in the GOP were joined together to exploit their spatial-temporal redundancies. In addition, the significant vectors of the joined sub-bands (within the GOP) were quantized using a multistage lattice vector quantization (MLVQ). For experimental purpose, TETRA Hilly Terrain (HT) environment was chosen, considering $15 \mathrm{fps}$ frame rate and QCIF resolution baseline profile. Further, to absorb the damaged video frames, equal and unequal error protection (EEP/UEP) scheme of the MLVQ for wireless channels was opted which uses Reed Solomon $(54,18)$ with coding rate of $1 / 3$. Moreover, a coding rate of $1 / 4$ was chosen for the header as it contains sensitive data. However, as texture contains low-band information, $2 / 3$ coding rate was used. This process reduced quantization errors and enhanced the reconstruction quality of the video frames using error-resilient tool. The study concludes that enhanced video codec performs better than H.263 on TETRA links. In [1], the authors explore the possibility of video over TETRA using the H.264/AVC video codec. Though, in [1], the authors considered video communications over TETRA release 2 but the channel capacities chosen for the experiments were not as per specifications provided by ETSI.

\subsection{TENS: A SIMULATION FRAMEWORK FOR TEDS}

To carry the proposed study, we propose a simulation framework, named TENS. TENS offers flexible, robust, and user friendly environment. TENS has implemented both "Voice plus data" and PDO protocol stacks using the specification and description language (SDL) to be translated using C code generator library. TENS offers a detailed user interface to enable users to select the desired scenarios, setting required parameters, and choosing appropriate algorithms. TENS is developed using C\#. Net and bases on TEDS protocol stack. The design of TENS is based on distributed system that optimizes both voice and packet data communication. Fig. 2 highlights the building blocks of TENS framework which 
includes simulation control entity, downlink/uplink channels, traffic generator, mobile station, base station, and propagation model. The interaction among modules is highlighted to illustrate the workflow.

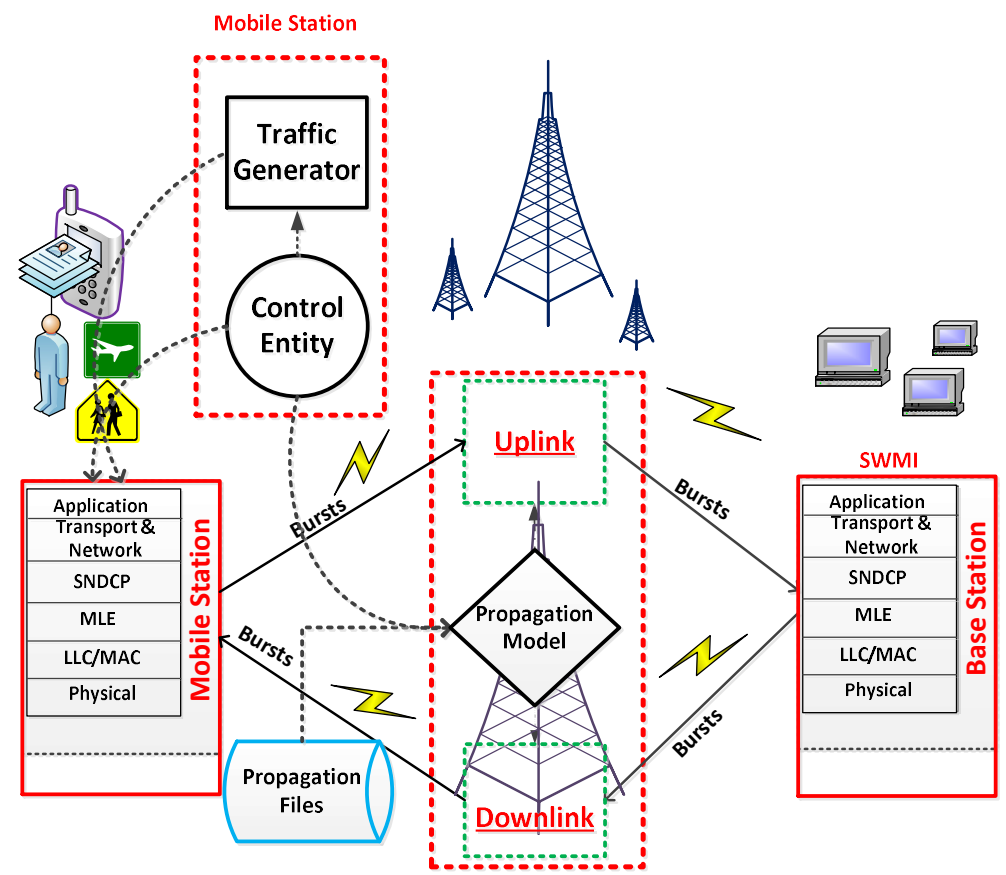

Fig. 2: TENS Simulation Framework Overview

Control entity, the main module within TENS framework, is responsible for controlling the whole simulation environment by configuring desired communicating entities. It controls traffic generator module and selects traffic patterns on the basis of application's demand. Traffic pattern entity simulates different simulation environments such as rural area and city environment to describe the communication environment. For video communication, multiple frame rates induce variable loads on the nodes. Moreover, control entity forms communication channels between other entities, where uplink/downlink channels module decides traffic flow direction, such as mobile to base station or base station to mobile communication. Furthermore, control entity controls propagation model according to the defined scenario. Different propagation environments can be accessed from the linked database to introduce particular noise on the channels as per demand of the simulation environment. The possible simulation scenarios includes: (i) medium density European city, (ii) typical rural public network, (iii) motorway linkage scenario, (iv) rescue operation scenarios to facilitate professionals such as police, medical services, fire brigade, and (v) city area network.

In TEDS layered architecture (see Fig. 2), TENS has opted RTP/UDP/IP convention for video communication, where RTP performs packet/frame handling at application layer [11,27]. Prior to accessing a Mobile Station (MS) to any SNDCP service, it first goes through a packet data registration procedure called PDP context activation that is initiated by the MS. An optional Multimedia Exchange (MEX) layer resides above SNDCP and routes the data to the destination [3]. On LLC, basic link offers traditional packet decomposition, while advanced link offers ACK (acknowledgment) service for reliable transmission $[3,15,19,28,29]$. In order to use TENS, user has to install it on numerous servers prior to switching on the particular mode (receiving or sending). For instance, in order to simulate mobile to SWMI communicating mode, the SWMI is tuned to receiving mode and MS is tuned to the sending mode of the TENS. 
To use TEDS channels, a TDMA-based approach is implemented which assigns time slots to the users to transfer data. Moreover, we use FCFS approach to access channels, and have implemented a scheduler which exploits 4 slotted TDMA approach to share TEDS channels as described in Algorithm 1(1 TDMA=14.56ms). TENS scheduler insures fair sharing of TEDS channels among mobile users based on 4 slotted TDMA frames. The mobile user waits for its turn to access the TEDS channel(s) and hold it until completion of its TDMA slot duration. Further, it divides SNDCP video frames into " $k$ " packets based on Maximum Transfer Unit (MTU) prior to transferring them during allotted frame slot on the selected channel. MTU is calculated based on chosen modulation scheme, modulation rate, and coding scheme for specified channels (see Table 1) at LLC layer.

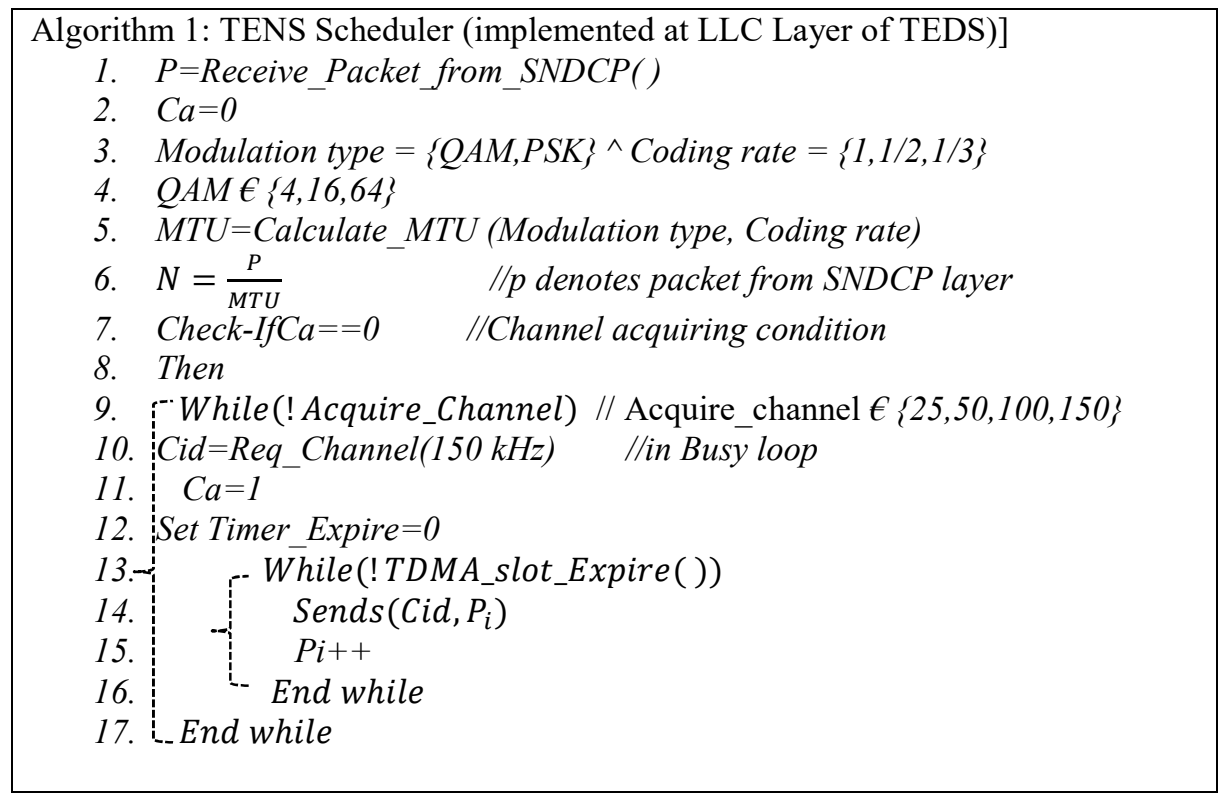

Table 2: Downlink/uplink channel capacities using different QAM modulation rates

\begin{tabular}{|l|l|l|c|c|c|c|}
\hline QAM & Bits/symbol & Coding Rate & \multicolumn{4}{|c|}{ Downlink/uplink (Bits per slot) } \\
\hline & & & $25 \mathrm{kHz}$ & $50 \mathrm{kHz}$ & $100 \mathrm{kHz}$ & $150 \mathrm{kHz}$ \\
\hline 4 & 2 & $1 / 2$ & $185 / 181$ & $421 / 389$ & $893 / 805$ & $1365 / 1221$ \\
\hline 16 & 4 & $1 / 2$ & $389 / 381$ & $861 / 797$ & $1805 / 1629$ & $2749 / 2461$ \\
\hline 16 & 4 & 1 & $800 / 784$ & $1744 / 1616$ & $3632 / 3280$ & $5520 / 4944$ \\
\hline 64 & 6 & $1 / 2$ & $593 / 581$ & $1301 / 1205$ & $2717 / 2453$ & $4133 / 3701$ \\
\hline 64 & 6 & $1 / 3$ & $797 / 781$ & $1741 / 1613$ & $3535 / 3277$ & $5517 / 4941$ \\
\hline 64 & 6 & 1 & $1208 / 1184$ & $2624 / 2432$ & $5456 / 4928$ & $3629 / 7424$ \\
\hline
\end{tabular}

In Table 2, channel capacities are given for all TEDS links. However, we carried our experimentations on $150 \mathrm{KHz}$ channel for uplink communication because it offers the highest channel capacity. Moreover, we have chosen QAM 64 modulation, 6 bits/carrier, and a coding rate of 1, to investigate the behaviour of TEDS technology for best case scenario. 


\subsection{EXPERIMENTS AND RESULTS}

This section discusses our experimental setup and findings.

\subsection{Experimental Setup}

The simulations are performed using the proposed TENS framework. To simulate mobile station to SWMI communication scenario, the framework was installed on two systems. For communications, both source and destination servers had almost similar computational and storage capabilities (1 GB RAM, Intel Core 2 duo Processor, Windows 7). We used the standard video sequences, namely "Tokyo Olympics" (ToL)1 and "Silence of Lamb" (SOL)2 to carry our simulation on TEDS links. Furthermore, considering TEDS' limited bandwidth, we chose the QCIF (176X144) frame resolution for both video codecs. We chose frame rates of 10, 12, and 15 frames per second (fps) to investigate the behaviour of codecs on TEDS network performance and application video quality. We selected baseline profile (for both codecs) as it requires the least network resources. The details of the chosen codecs and video features are given in the Table 3 .

Table 3: Video codecs simulation parameters

\begin{tabular}{|l|l|l|}
\hline Parameters & \multicolumn{1}{|c|}{ MPEG-4 } & \multicolumn{1}{c|}{ H.264/AVC } \\
\hline Source format & DVD & DVD \\
\hline Video length & 30 minutes & 30 minutes \\
\hline Gop Size & 16 & 16 \\
\hline Mean Frame Size & $23908(\mathrm{ToL}), 15531(\mathrm{SoL})$ & $15259(\mathrm{ToL}), 7885(\mathrm{SoL})$ \\
\hline Profile & Baseline & Baseline \\
\hline Frames Sequence & G16B1 & G16B1 \\
\hline
\end{tabular}

For experimentation, we chose $150 \mathrm{KHz}$ channel as we were interested to see the maximum video quality that TEDS can offer to the PSS personnel. Furthermore, signal modulation with 2/3 (RCPC) channel code for TU 50 propagation environment is employed to represent the realistic environment. The performance of both codecs was studied against $2 \%, 5 \%$, and $10 \%$ packet loss. As TEDS "Bandwidth on Demand" feature enables the users to combine slots to increase throughput, we implemented this feature (Bandwidth on Demand) assuming that all TDMA frame slots are available to a single user.

\subsection{Measurement Parameters}

The section defines parameters that are used to investigate performance behaviour of video codecs against TEDS links.

\subsubsection{Peak Signal to Noise Ratio (PSNR)}

PSNR is the ratio of maximum power of signal to the noise. Equation 1 illustrates the measures of PSNR at the receiver' send $[\underline{5}, \underline{7}, \underline{19}, \underline{30}, \underline{31]}$.

$$
\operatorname{PSNR}=20 \log \frac{L}{\sqrt{M S E}}
$$

${ }^{1}$ http://trace.eas.asu.edu

${ }^{2}$ http://trace.eas.asu.edu 
Where,

$$
\operatorname{MSE}=\frac{1}{M N} \sum_{Y=1}^{M} \sum_{x=1}^{N}[I(X, Y)-R(X, Y)]
$$

In Equation 1, "L" is a constant that represents the range of pixel intensities, i.e. $L=255$ for 8-bit grey level image or a video frame, $\mathrm{I}(\mathrm{X}, \mathrm{Y})$ and $\mathrm{R}(\mathrm{X}, \mathrm{Y})$ represent original and approximated image, and $\mathrm{MxN}$ represents dimensions of a video frame.

\subsubsection{End-to-End Delay}

Video frames end-to-end delay defines the time duration a frame takes to reach the destination mobile from source. It is calculated by subtracting video frame sending time $\left(\mathrm{P}_{\mathrm{s}}\right)$ from the arrival time $\left(\mathrm{P}_{\mathrm{r}}\right)$ as shown in Equation 2.

$$
\mathrm{D}=P_{r}-P_{s}
$$

\subsubsection{Frame Drop Rate}

The application frame drop ratio is the ratio of total video frames received $(\mathrm{N})$ to the sent $(\mathrm{k})$ and can be calculated using Equation 3.

$$
\text { Received }(\%)=\frac{\mathrm{N}}{\mathrm{k}}
$$

The frame drop (in percentage) rate is calculated using Equation 4.

$$
\text { Frame Drop Rate }(\%)=100-\text { Received }
$$

\subsection{Statistical Analysis}

To check the significance of the results, we used Statistical Package of Social Sciences (SPSS) 20.0 [9] for data analysis. Paired sample t-test (2-tailed) was applied to verify whether the change (end-to-end delay, frame drop, and PSNR) incurred by both codecs is significant.

The hypotheses for statistical analysis of the results are:

- Null hypothesis

$\mathrm{H}_{0}: \mu_{1}=\mu_{2}\left(\mathrm{H}_{0}\right.$ : The end-to-end delay, PSNR and frame drop of both codecs is equal)

- Alternate hypothesis

$\mathrm{H}_{1}: \mu_{1}<\mu_{2}$

$\mathrm{H}_{2}: \mu_{1}>\mu_{2}$

The frame drop, end-to-end delay, and PSNR of both codecs are not equal.

- Significance level

$\alpha=0.05(5 \%)$

- Decision rule

If $\mathrm{P}<\alpha$ then Reject $\mathrm{H}_{0}$

If $\mathrm{t}>0$, first codec performs better

If $\mathrm{t}<0$, second codec performs better

Else Accept $\mathrm{H}_{0}$ 


\subsection{Results and Discussion}

This section discusses TEDS network behaviour and reconstructed video quality transferred using H.264/AVC and MPEG-4 encoded video sequences. We evaluate the performance of H.264/AVC and MPEG-4 against several channel propagation conditions and video frame rates. Furthermore, we analyse the behaviour of TEDS for multiple video patterns using H.264/AVC encoded video streams.

\subsubsection{Codecs Performance Comparison}

This section highlights the behaviour of H.264/AVC and MPEG-4 video codecs by passing TOL video sequence. Fig.3, Fig.4, and Fig.5 present average end-to-end delay against three different frame rates and varying channel propagation conditions. It is evident that MPEG-4 incurs higher end-to-end delays compared to H.264/AVC encoded video sequence. Moreover, the end-to-end delay increases along the increase in the frame rate of the video. To analyse the difference between the end-to-end delays of the codecs, we performed a statistical analysis (see Table 4, Table 5, and Table 6). The statistics analysis shows there is insignificant difference (i.e., $P>\alpha$ ) between the end-to-end delays of the selected codecs for 10,12 , and $15 \mathrm{fps}$ with packet loss of $2 \%, 5 \%$, and $10 \%$, respectively.

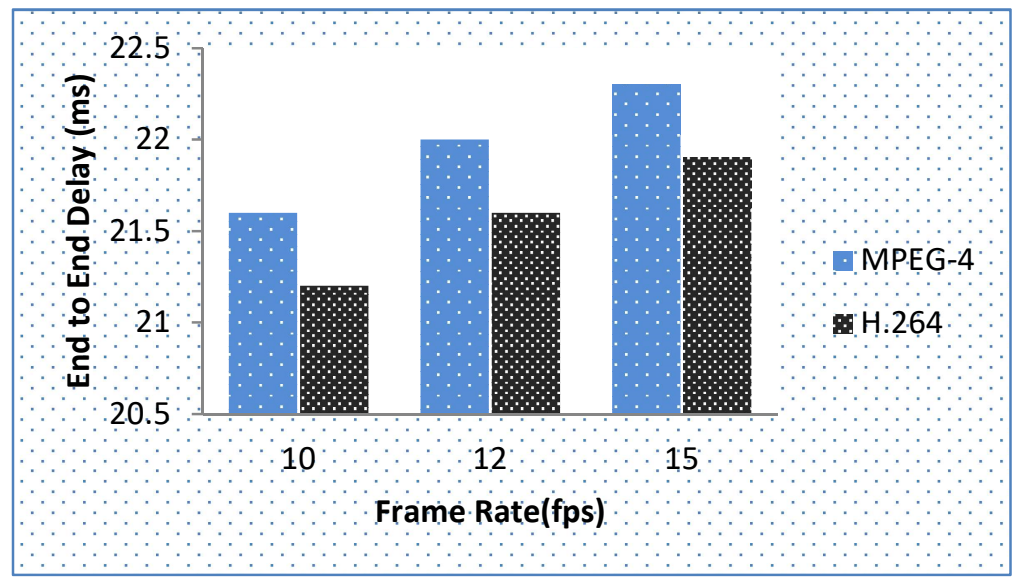

Fig. 3: Avg. end-to-end delay for $2 \%$ packet loss

Table 4: Statistical analysis of end-to-end delay at $2 \%$ packet loss

\begin{tabular}{|l|l|l|l|l|l|l|}
\hline & \multicolumn{2}{|c|}{ 10 FPS } & \multicolumn{2}{c|}{ 12 FPS } & \multicolumn{2}{c|}{ 15 FPS } \\
\hline & H.264 AVC & MPEG 4 & H.264 AVC & MPEG-4 & H.264 AVC & MPEG-4 \\
\hline Mean & 21.3000 & 21.6200 & 21.6800 & 22.0700 & 21.9400 & 22.3700 \\
\hline Std. Deviation & 3.55371 & 2.55639 & 1.66320 & 1.60558 & 2.36934 & 1.53119 \\
\hline Std. Error Mean & 1.12378 & .80840 & .52595 & .50773 & .74925 & .48421 \\
\hline N & 10 & & 10 & & 10 & \\
\hline Mean Difference & -.32000 & & -.39000 & & -.43000 & \\
\hline Std. Deviation & 4.97255 & & .81028 & & 2.48598 & \\
\hline Std. Error Mean & 1.57246 & & .25623 & & .78614 & \\
\hline Lower & -3.87715 & & -.96964 & & -2.20837 & \\
\hline Upper & 3.23715 & & .18964 & & 1.34837 & \\
\hline T & -.204 & & -1.522 & & -.547 & \\
\hline Df & 9 & & 9 & & 9 & \\
\hline Sig. (2-tailed) & .843 & & .162 & & .598 & \\
\hline
\end{tabular}




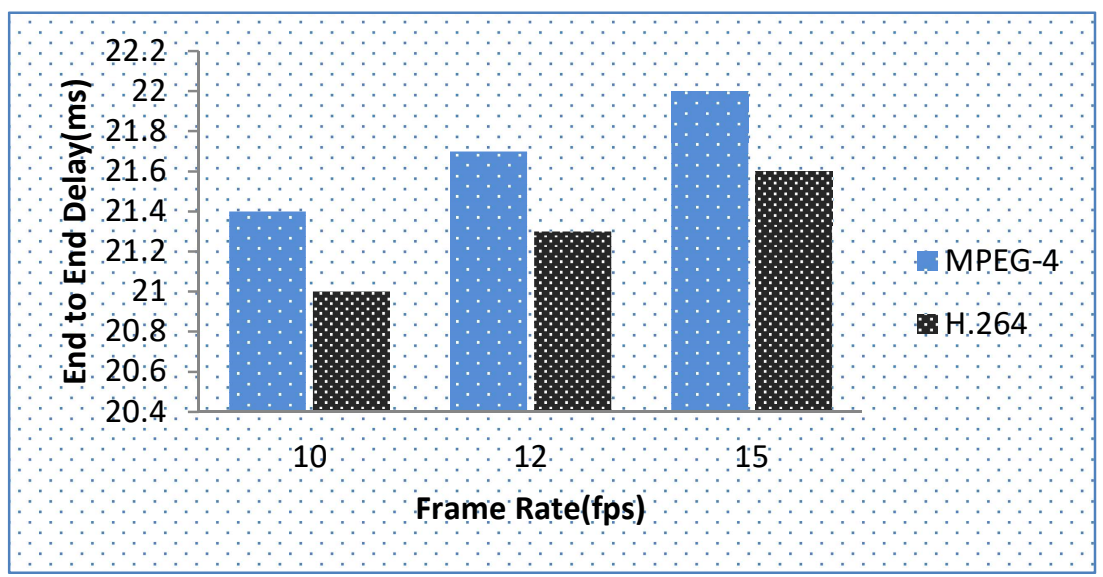

Fig. 4: Avg. end-to-end delay for 5\% packet loss

Table 5: Statistical analysis of end-to-end delay at 5\% packet loss

\begin{tabular}{|l|l|l|l|l|l|l|}
\hline & \multicolumn{2}{|c|}{ 10 FPS } & \multicolumn{2}{c|}{ 12 FPS } & \multicolumn{2}{c|}{ 15 FPS } \\
\hline & H.264 AVC & MPEG-4 & H.264 AVC & MPEG-4 & H.264 AVC & MPEG-4 \\
\hline Mean & 21.1000 & 21.4500 & 21.4100 & 21.7200 & 21.6400 & 22.1500 \\
\hline Std. Deviation & 1.93506 & 1.50573 & 1.80398 & 1.63558 & 1.41437 & 1.86503 \\
\hline Std. Error Mean & .61192 & .47615 & .57047 & .51721 & .44726 & .58977 \\
\hline $\mathrm{N}$ & 10 & & 10 & & 10 & \\
\hline Mean Difference & -.35000 & & -.31000 & & -.51000 & \\
\hline Std. Deviation & 2.59197 & & 1.24226 & & 2.08990 & \\
\hline Std. Error Mean & .81965 & & .39284 & & .66088 & \\
\hline Lower & -2.20419 & & -1.19866 & & -2.00502 & \\
\hline Upper & 1.50419 & & .57866 & & .98502 & \\
\hline t & -.427 & & -.789 & & -.772 & \\
\hline Df & 9 & & 9 & & 9 & \\
\hline Sig. (2-tailed) & .679 & & .450 & & .460 & \\
\hline
\end{tabular}


For lower Mean Error Rate (MER), the average end-to-end delay is relatively high for the reason that end-to-end delay depends on the frame size. TENS uses UDP as transport layer protocol which transfers video frame to the application if all video frame chunks are received. In addition, due to lower probability of packet dropping at LLC, lower MER increases the size of video frames that reach the destination. Consequently, the average end-to-end delay is higher. Moreover, with $10 \%$ MER, the end-to-end delay is smaller, as compared to $2 \%$ and $5 \%$ packet loss due to the UDP implementation at the transport layer.

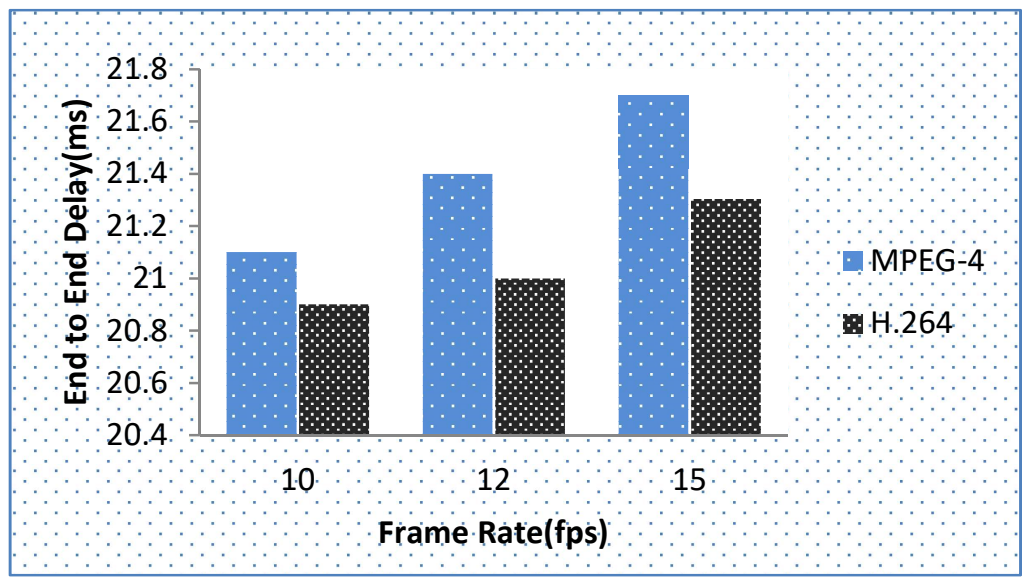

Figure 5: Avg. end-to-end delay for 10\% packet loss

Table 6: Statistical analysis of end-to-end delay at $10 \%$ packet loss

\begin{tabular}{|l|l|l|l|l|l|l|}
\hline & \multicolumn{2}{|c|}{ 10 FPS } & \multicolumn{2}{c|}{ 12 FPS } & \multicolumn{2}{c|}{ 15 FPS } \\
\hline & H.264 AVC & MPEG-4 & H.264 AVC & MPEG-4 & H.264 AVC & MPEG-4 \\
\hline Mean & 20.9200 & 21.2100 & 21.0100 & 21.3600 & 21.2200 & 21.7600 \\
\hline Std. Deviation & 1.65113 & 1.47381 & .96661 & 1.50643 & 4.20735 & 1.13353 \\
\hline Std. Error Mean & .52213 & .46606 & .30567 & .47638 & 1.33048 & .35845 \\
\hline $\mathrm{N}$ & 10 & & 10 & & 10 & \\
\hline Mean Difference & -.29000 & & -.35000 & & -.54000 & \\
\hline Std. Deviation & .47011 & & .78351 & & 3.90134 & \\
\hline Std. Error Mean & .14866 & & .24777 & & 1.23371 & \\
\hline Lower & -.62629 & & -.91049 & & -3.33085 & \\
\hline Upper & .04629 & & .21049 & & 2.25085 & \\
\hline $\mathrm{t}$ & -1.951 & & -1.413 & & -.438 & \\
\hline Df & 9 & & 9 & & 9 & \\
\hline Sig. (2-tailed) & .083 & & .191 & & .672 & \\
\hline
\end{tabular}


At LLC, TENS holds incoming video frames in storage (buffer) unless a channel slot is available for the packets. In addition, MTU allows limited amount of data to be transferred in a time unit. Consequently, for higher frame rate, the average end-to-end delay time is higher. In contrast, lower frame rate introduces lower average end-to-end delays due to smaller buffering delay time. Hence, for $15 \mathrm{fps}$, the end-to-end delay is higher as compared to 2 and 5 fps. Fig.6, Fig.7, and Fig. 8 show that higher packet drop rate at physical layer leads to higher application video frame loss rate. The statistical data presented in Table 7, Table 8, and Table 9 show that the difference between the frame drop rate of the codecs is significant (i.e., $\mathrm{P}<\alpha$ ) for all occurrences except for $10 \mathrm{fps}$ (with $2 \%$ packet loss) and $15 \mathrm{fps}$ (with $10 \%$ packet loss).

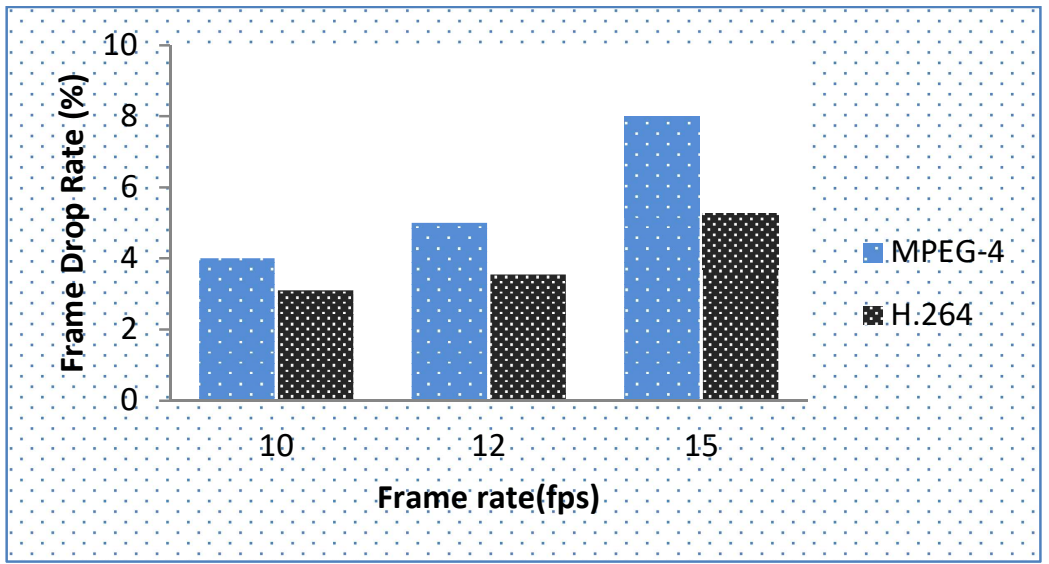

Fig. 6: Avg. Video frames drop rate at $2 \%$ packet loss

Table 7: Statistical analysis of video frames drop rate at $2 \%$ packet loss

\begin{tabular}{|l|l|l|l|l|l|l|}
\hline & \multicolumn{2}{|c|}{10 FPS } & \multicolumn{2}{c|}{ 12FPS } & \multicolumn{2}{c|}{ 15FPS } \\
\hline & H.264 AVC & MPEG-4 & H.264 AVC & MPEG-4 & H.264 AVC & MPEG-4 \\
\hline Mean & 3.0600 & 3.9900 & 3.4500 & 5.1300 & 5.2700 & 8.0200 \\
\hline Std. Deviation & .91190 & .75638 & .81138 & .99783 & .95922 & .92592 \\
\hline Std. Error Mean & .28837 & .23919 & .25658 & .31554 & .30333 & .29280 \\
\hline $\mathrm{N}$ & 10 & & 10 & & 10 & \\
\hline Mean Difference & -.93000 & & -1.68000 & & -2.75000 & \\
\hline Std. Deviation & 1.34994 & & 1.06124 & & 1.50499 & \\
\hline Std. Error Mean & .42689 & & .33559 & & .47592 & \\
\hline Lower & -1.89569 & & -2.43916 & & -3.82661 & \\
\hline Upper & .03569 & & -.92084 & & -1.67339 & \\
\hline $\mathrm{t}$ & -2.179 & & -5.006 & & -5.778 & \\
\hline Df & 9 & & 9 & & 9 & \\
\hline Sig. (2-tailed) & .057 & & .001 & & .000 & \\
\hline
\end{tabular}




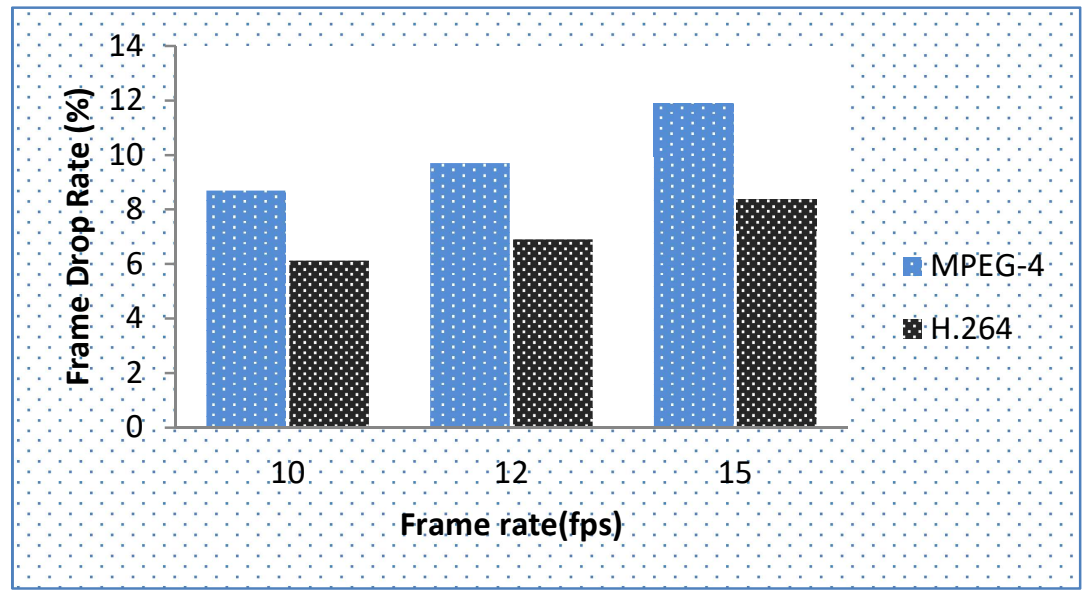

Fig. 7: Avg. video frames drop at 5\% packet loss

Table 8: Statistical analysis of video frames drop at 5\% packet loss

\begin{tabular}{|l|l|l|l|l|l|l|}
\hline & \multicolumn{2}{|c|}{ 10 FPS } & \multicolumn{2}{c|}{ 12 FPS } & \multicolumn{2}{c|}{ 15 FPS } \\
\hline & H.264 AVC & MPEG-4 & H.264 AVC & MPEG-4 & H.264 AVC & MPEG-4 \\
\hline Mean & 6.1400 & 8.7100 & 6.8500 & 9.7000 & 8.2500 & 11.8500 \\
\hline Std. Deviation & 1.20204 & 1.07129 & 1.21678 & 1.29099 & .77064 & 1.98116 \\
\hline Std. Error Mean & .38012 & .33877 & .38478 & .40825 & .24370 & .62650 \\
\hline $\mathrm{N}$ & 10 & & 10 & & 10 & \\
\hline Mean Difference & -2.57000 & & -2.85000 & & -3.60000 & \\
\hline Std. Deviation & 1.60073 & & 2.29359 & & 2.37767 & \\
\hline Std. Error Mean & .50619 & & .72530 & & .75189 & \\
\hline Lower & -3.71509 & & -4.49074 & & -5.30089 & \\
\hline Upper & -1.42491 & & -1.20926 & & -1.89911 & \\
\hline T & -5.077 & & -3.929 & & -4.788 & \\
\hline Df & 9 & & 9 & & 9 & \\
\hline Sig. (2-tailed) & .001 & & .003 & & .001 & \\
\hline
\end{tabular}

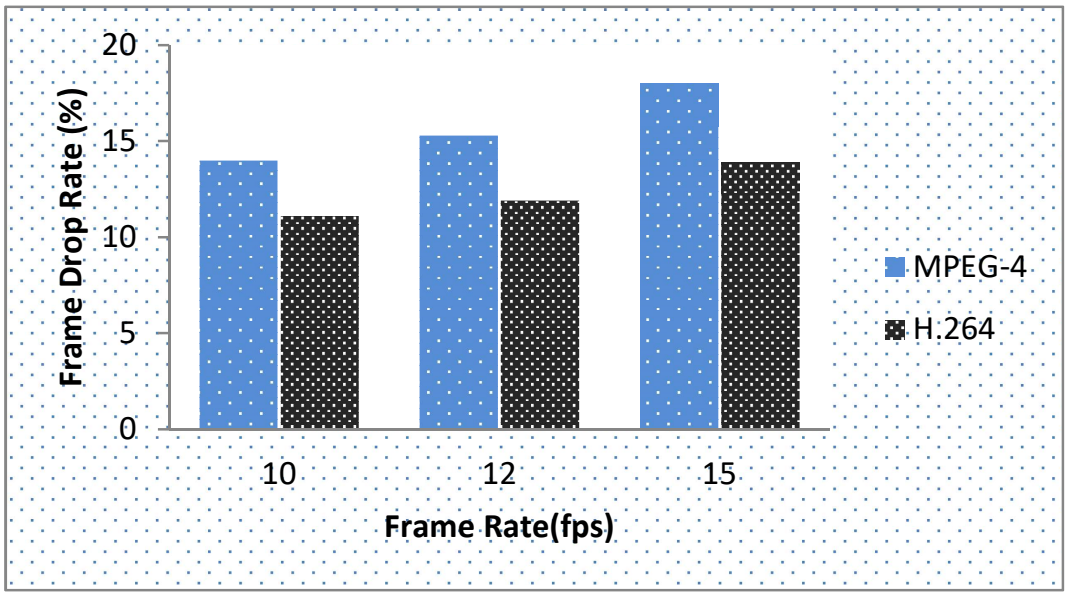

Fig. 8: Avg. video frames drop rate at $10 \%$ packet loss 
Table 9: Statistical analysis of video frames drop rate at 10\% packet loss

\begin{tabular}{|l|l|l|l|l|l|l|}
\hline & \multicolumn{2}{|c|}{ 10 FPS } & \multicolumn{2}{c|}{ 12 FPS } & \multicolumn{2}{c|}{ 15 FPS } \\
\hline & H.264 AVC & MPEG-4 & H.264 AVC & MPEG-4 & H.264 AVC & MPEG-4 \\
\hline Mean & 11.0500 & 14.0200 & 11.9000 & 15.3100 & 13.8200 & 18.0200 \\
\hline Std. Deviation & 1.24030 & 2.36962 & 1.19443 & 1.82906 & 1.42657 & 1.61644 \\
\hline Std. Error Mean & .39222 & .74934 & .37771 & .57840 & .45112 & .51116 \\
\hline N & 10 & & 10 & & 10 & \\
\hline Mean Difference & -2.97000 & & -3.41000 & & -4.20000 & \\
\hline Std. Deviation & 2.85387 & & 2.56751 & & 2.66833 & \\
\hline Std. Error Mean & .90247 & & .81192 & & .84380 & \\
\hline Lower & -5.01153 & & -5.24669 & & -6.10881 & \\
\hline Upper & -.92847 & & -1.57331 & & -2.29119 & \\
\hline T & -3.291 & & -4.200 & & -4.977 & \\
\hline Df & 9 & & 9 & & 9 & \\
\hline Sig. (2-tailed) & .009 & & .002 & & .001 & \\
\hline
\end{tabular}

It is observed that with $\mathrm{x} \%$ packet drop rate at LLC, $(\mathrm{x}+\mathrm{k}) \%$ video frames are dropped, where " $\mathrm{k}$ " is an integer. UDP at transport layer is one of the major reasons for the extra "k" video frame drop. In case of $2 \%$ MER, less number of frames is dropped, resultantly; the video frame drop rate at application is lower as compared to the 5\% and $10 \%$ MER. In case of H.264/AVC, the application video frame drop rate is lower as compared to the MPEG-4 due to smaller video frame size. As frame rate relies on the bandwidth, the increase in frame rate increases video frame drop rate at the application layer. As shown in Fig.6, Fig.7, and Fig.8, at $15 \mathrm{fps}$ video, video frame drop rate is higher compared to 2 and 5 fps. Table 7, Table 8, and Table 9 show that the difference between the video frame drop rate of the codecs is significant (i.e., $\mathrm{P}<\alpha$ ) for all occurrences except for $10 \mathrm{fps}$ (with $2 \%$ packet loss) and $15 \mathrm{fps}$ (with $10 \%$ packet loss). Fig.9, Fig.10, and Fig.11 show measures of PSNR against several codec rates and channel conditions. Further, Table 10, Table 11, and Table 12 highlight the difference between PSNR while using diversified codec rates.

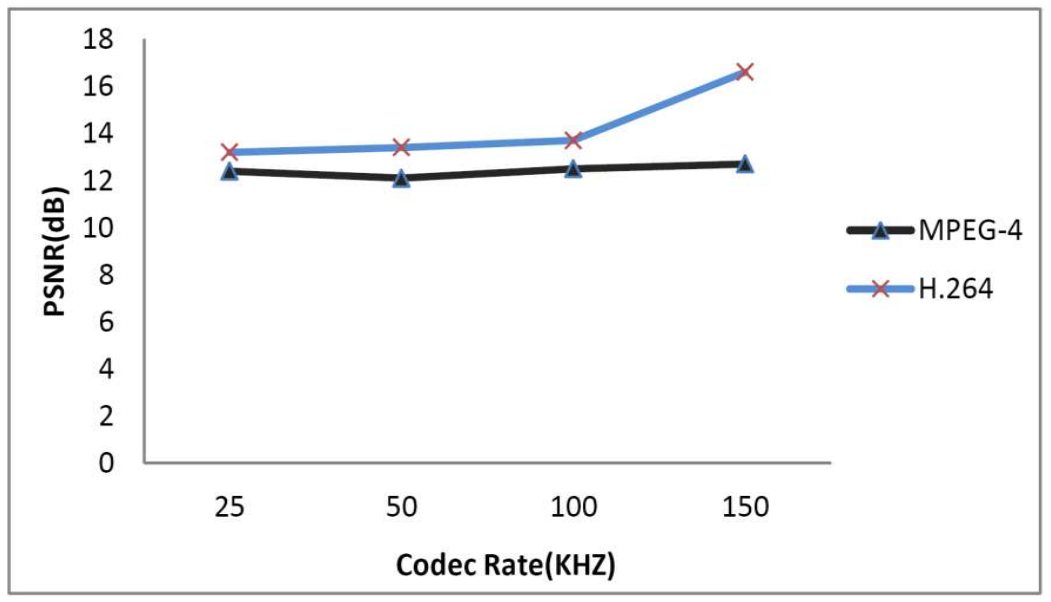

Figure 9: H.264/AVC and MPEG-4PSNR comparison at 2\% packet loss 
Table 10: Statistical analysis of PSNR at $2 \%$ packet loss

\begin{tabular}{|l|l|l|l|l|l|l|l|l|}
\hline & \multicolumn{2}{|c|}{$25 \mathrm{kHz}$} & \multicolumn{2}{c|}{$50 \mathrm{kHz}$} & \multicolumn{2}{c|}{$100 \mathrm{kHz}$} & \multicolumn{2}{c|}{$150 \mathrm{kHz}$} \\
\hline & $\begin{array}{l}\text { H.264 } \\
\text { AVC }\end{array}$ & MPEG-4 & $\begin{array}{l}\text { H.264 } \\
\text { AVC }\end{array}$ & $\begin{array}{l}\text { MPEG- } \\
4\end{array}$ & $\begin{array}{l}\text { H.264 } \\
\text { AVC }\end{array}$ & $\begin{array}{l}\text { MPEG- } \\
4\end{array}$ & $\begin{array}{l}\text { H.264 } \\
\text { AVC }\end{array}$ & $\begin{array}{l}\text { MPEG- } \\
4\end{array}$ \\
\hline Mean & 13.1500 & 12.4400 & 13.3500 & 12.1300 & 13.6500 & 12.5400 & 16.5800 & 12.6500 \\
\hline Std. Deviation & 1.29207 & 1.15200 & 1.59948 & 1.21842 & 1.67680 & 1.71153 & 1.60333 & 1.57639 \\
\hline Std. Error Mean & .40859 & .36430 & .50580 & .38530 & .53025 & .54123 & .50702 & .49850 \\
\hline N & 10 & & 10 & & 10 & & 10 & \\
\hline Mean Difference & .71000 & & 1.22000 & & 1.11000 & & 3.93000 & \\
\hline Std. Deviation & 2.04909 & & 2.13687 & & 1.96041 & & 1.97262 & \\
\hline Std. Error Mean & .64798 & & .67574 & & .61994 & & .62380 & \\
\hline Lower & -.75583 & & -.30863 & & -.29240 & & 2.51887 & \\
\hline Upper & 2.17583 & & 2.74863 & & 2.51240 & & 5.34113 & \\
\hline T & 1.096 & & 1.805 & & 1.791 & & 6.300 & \\
\hline Df & 9 & & 9 & & 9 & & 9 & \\
\hline Sig. (2-tailed) & .302 & & .104 & & .107 & & .000 & \\
\hline
\end{tabular}

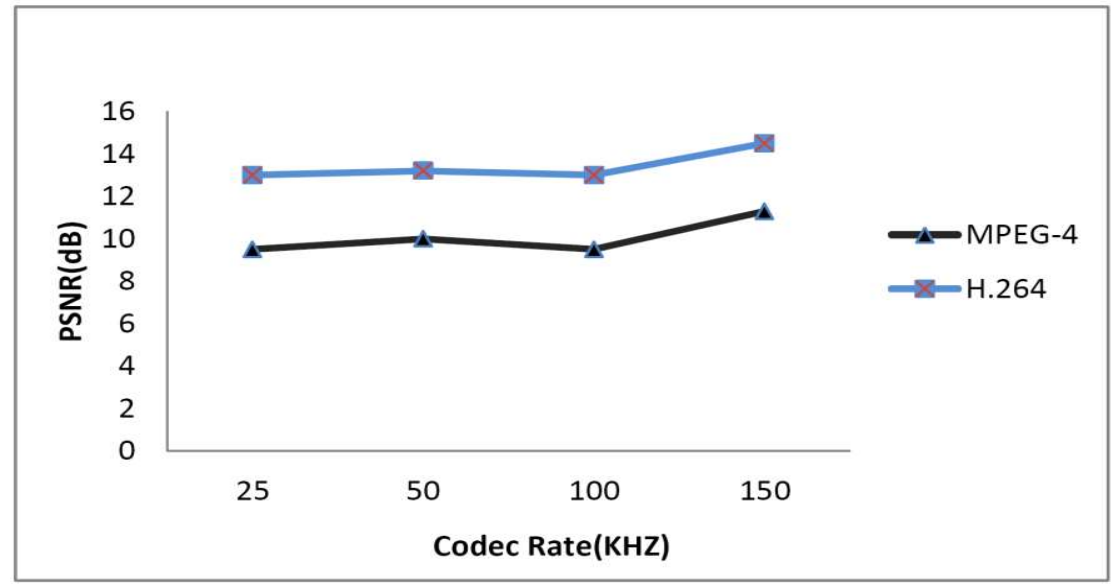

Fig. 10: PSNR comparison among H.264/AVC and MPEG-4 for 5\% packet loss 
Table 11: Statistical analysis of PSNR at $5 \%$ packet loss

\begin{tabular}{|l|l|l|l|l|l|l|l|l|}
\hline & \multicolumn{2}{|c|}{$25 \mathrm{kHz}$} & \multicolumn{2}{c|}{$50 \mathrm{kHz}$} & \multicolumn{2}{c|}{$100 \mathrm{kHz}$} & \multicolumn{2}{c|}{$150 \mathrm{kHz}$} \\
\hline & $\begin{array}{l}\text { H.264 } \\
\text { AVC }\end{array}$ & MPEG-4 & H.264 AVC & MPEG-4 & H.264 AVC & MPEG-4 & H.264 AVC & MPEG-4 \\
\hline Mean & 12.9500 & 9.4800 & 13.1800 & 10.0300 & 13.0000 & 9.4500 & 14.5200 & 11.2100 \\
\hline Std. Deviation & 1.42302 & 1.27000 & 1.94182 & 1.39368 & 1.69967 & .99917 & 1.66986 & .84781 \\
\hline Std. Error Mean & .45000 & .40161 & .61406 & .44072 & .53748 & .31596 & .52806 & .26810 \\
\hline N & 10 & & 10 & & 10 & & 10 & \\
\hline Mean Difference & 3.47000 & & 3.15000 & & 3.55000 & & 3.31000 & \\
\hline Std. Deviation & 1.93681 & & 1.92137 & & 1.60503 & & 1.73041 & \\
\hline Std. Error Mean & .61247 & & .60759 & & .50755 & & .54721 & \\
\hline Lower & 2.08449 & & 1.77553 & & 2.40183 & & 2.07214 & \\
\hline Upper & 4.85551 & & 4.52447 & & 4.69817 & & 4.54786 & \\
\hline T & 5.666 & & 5.184 & & 6.994 & & 6.049 & \\
\hline Df & 9 & & 9 & & 9 & & 9 & \\
\hline Sig. (2-tailed) & .000 & & .001 & & .000 & & .000 & \\
\hline
\end{tabular}



Fig. 11: PSNR comparison between H.264/AVC and MPEG-4 for 10\% packet loss

We noticed that higher packet drop rate minimizes the observed PSNR value, and the type of video frame dropped during video communication affects the PSNR value. Video frames are classified as I, P and B frames, as discussed in Section 4.2. "I" frames are more important than "P" and "B" frames during the decoding process because "P" and "B" are decoded based on "I" frame. Therefore, if more "I" video frames get corrupted during the video transfer phase, then the video quality suffers badly. For H.264/AVC, video quality in terms of PSNR value is good because lesser packets are dropped at LLC as compared to MPEG-4. Moreover, as error recovery feature of H.264/AVC is more powerful than MPEG-4, H.264/AVC offers better video quality on TEDS channels. However, the video quality is still poor, because only a video that has PSNR value greater than 25 is considered a good quality video. 
Table 12: Statistical analysis of PSNR at 10\% packet loss

\begin{tabular}{|l|l|l|l|l|l|l|l|l|}
\hline & \multicolumn{2}{|c|}{$25 \mathrm{kHz}$} & \multicolumn{2}{c|}{$50 \mathrm{kHz}$} & \multicolumn{2}{c|}{$100 \mathrm{kHz}$} & \multicolumn{2}{c|}{$150 \mathrm{kHz}$} \\
\hline & $\begin{array}{l}\text { H.264 } \\
\text { AVC }\end{array}$ & $\begin{array}{l}\text { MPEG- } \\
4\end{array}$ & $\begin{array}{l}\text { H.264 } \\
\text { AVC }\end{array}$ & $\begin{array}{l}\text { MPEG- } \\
4\end{array}$ & $\begin{array}{l}\text { H.264 } \\
\text { AVC }\end{array}$ & $\begin{array}{l}\text { MPEG- } \\
4\end{array}$ & $\begin{array}{l}\text { H.264 } \\
\text { AVC }\end{array}$ & $\begin{array}{l}\text { MPEG- } \\
4\end{array}$ \\
\hline Mean & 12.9500 & 9.8800 & 12.9800 & 9.5400 & 12.5100 & 8.5500 & 11.7200 & 8.3900 \\
\hline Std. Deviation & .98460 & .95429 & 2.00155 & .67198 & 1.82602 & .54006 & 1.52956 & .64713 \\
\hline Std. Error Mean & .31136 & .30177 & .63295 & .21250 & .57744 & .17078 & .48369 & .20464 \\
\hline $\mathrm{N}$ & 10 & & 10 & & 10 & & 10 & \\
\hline Mean Difference & 3.07000 & & 3.44000 & & 3.96000 & & 3.33000 & \\
\hline Std. Deviation & 1.12748 & & 2.27019 & & 2.02605 & & 1.41347 & \\
\hline Std. Error Mean & .35654 & & .71790 & & .64069 & & .44698 & \\
\hline Lower & 2.26345 & & 1.81600 & & 2.51065 & & 2.31887 & \\
\hline Upper & 3.87655 & & 5.06400 & & 5.40935 & & 4.34113 & \\
\hline T & 8.610 & & 4.792 & & 6.181 & & 7.450 & \\
\hline Df & 9 & & 9 & & 9 & & & 9 \\
\hline Sig. (2-tailed) & .000 & & .001 & & .000 & & .000 & \\
\hline
\end{tabular}

\subsubsection{Performance Evaluation of Multimedia Applications on TEDS}

This section discusses effects of different video contents on TEDS behaviour. For this experiment, we considered two video sequences, and H.264/AVC video codec. The results show that the video quality is degraded when high motion video contents are transferred over TEDS links. This behaviour is mainly due to the TEDS bandwidth limitations and abrupt video scene changes. On the other hand, for a moderate content video, TEDS performs well and the video quality is slightly affected. In these experiments, we considered 5\% packet loss rate as a standard. We used notation "A" and " $\mathrm{B}$ " to represent two videos, where video " $\mathrm{A}$ " is more stable as compared to video " $\mathrm{B}$ ". More specifically, video " $\mathrm{B}$ " is a drama serial (silence of lamb) 3 and video "A" (Tokyo Olympics)4 is a live football match having higher motion as compared to former. Fig. 12 plots the average end-to-end delay between two videos against several frame rates using H.264/AVC codec. As illustrated, video "A" outperforms video "B" due to high motion scenes in video "B". Also, the effect of frame rate on end-to-end delay is very minor. During higher frame rates, many large size (bulky) frames get corrupt at the physical layer due to UDP implementation and buffer timeouts. The bulky frames are mainly caused by an abrupt video scene change in a high motion video and may incur high end-to-end delays on low bandwidth networks (see Fig. 13).

\footnotetext{
${ }^{3}$ http://trace.eas.asu.edu/h264/
}

${ }^{4}$ http://trace.eas.asu.edu/h264/ 


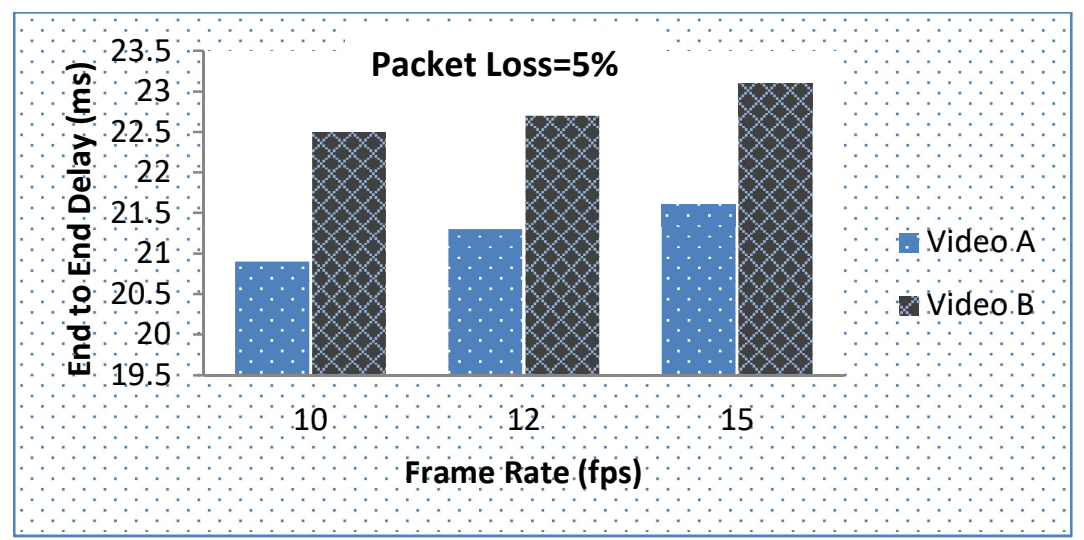

Figure 12: Avg. end-to-end delay comparison between Video "A" and "B"

Fig.13 presents frame drop rate using H.264/AVC video codec. It is evident that video "A" incurs less packet drop as compared to video "B". Moreover, the packet drop rate increases along the increase in the frame rate. It is because higher frame rates rely on the bandwidth capacity and may cause increased video frame drop as compared to lower frame rates. Fig. 14 presents the PSNR values for low and high motion videos.

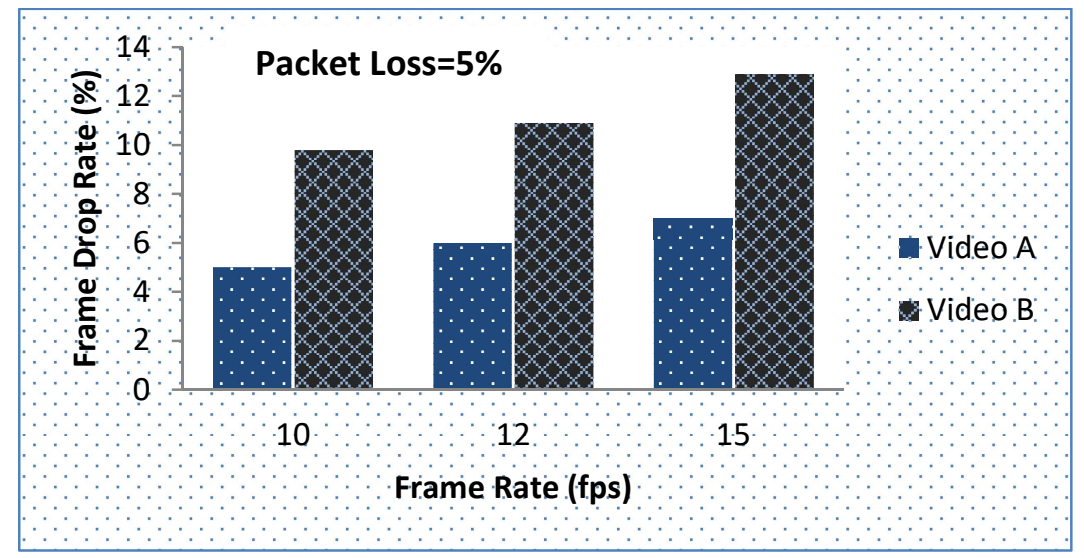

Fig. 13: Avg. frame drop rate comparison of Video "A" and "B"

It is evident that for both videos, PSNR increases along the increase in the codec rate. Moreover, the PSNR of video "A" is high, as compared to video "B". It is because the frame drop rate of video "B" is high as compared to video "A", which ultimately affects the quality of reconstructed video at the receiver's end. In addition, due to lower bandwidth of the TEDS channels, the information frame (I) loss rate of video "B" is lower as compared to video "A". 


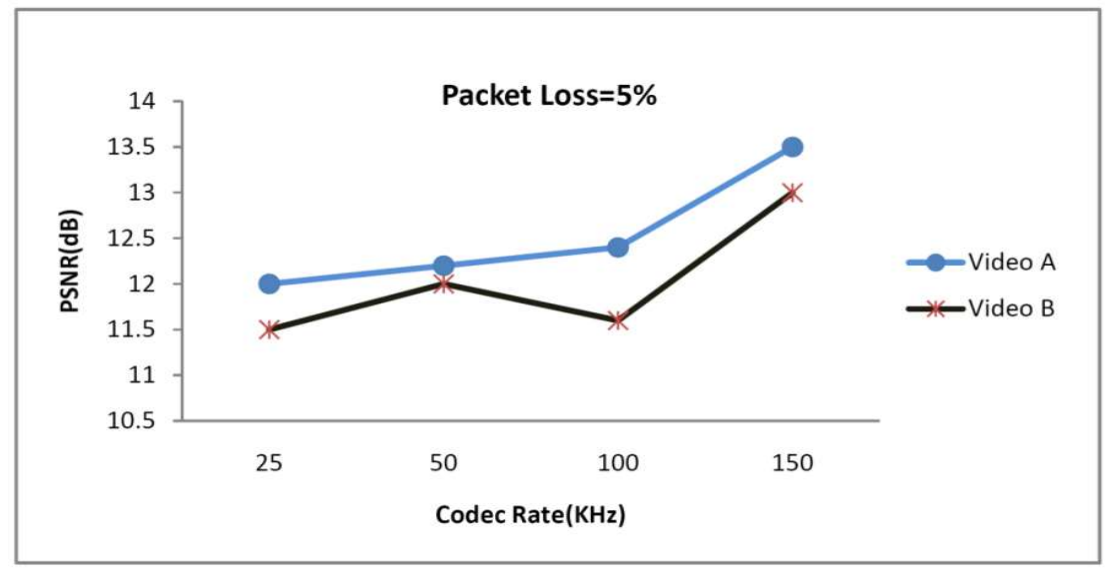

Fig. 3: Avg. PSNR comparison of Video "A" and "B"

\subsection{Observations \& Proposed Applications}

Video communication over low bandwidth networks is a challenging task due to intermittent network connectivity. Furthermore, transferring large-sized video frames significantly affects video quality and network performance [32, 33]. Based on the aforementioned discussion, we have proposed a few suitable applications/services for TETRA which are listed as follows:

- Telnet: Telnet services demands lower end-to-end delay and limited bandwidth capacity. It performs data transfer between the sender and receiver in form of strings/characters and requires delay of less than 250 milliseconds (ms). As TETRA's bandwidth is sufficient for the telnet required bandwidth (i.e., $1 \mathrm{kbps}$ ), it can be used for non-real time and asymmetric communications.

- Internet Relay Chat (IRC): In this service, multiple users communicate with one another in private or group form. It requires approximately $1 \mathrm{kbps}$ bandwidth and allows at most $200 \mathrm{~ms}$ delays. As the requirements of IRC can be fulfilled by TETRA, it can be an effective application for this technology.

- Audio Broadcasting: In audio conferencing, user response time is very important. It is measured as the time elapsed between transmitting command and response time. Audio conferencing demands delay $<150 \mathrm{~ms}$, and bandwidth of 60-80 kbps. Therefore, Voice over IP (VoIP) service requirements is also within range of TETRA specifications.

- Video Broadcasting: Video conferencing is suitable for delays $<150 \mathrm{~ms}$, and a jitter $<100 \mathrm{~ms}$, but it demands high bandwidth. Hence, it is possible to broadcast a low quality video over TEDS channels.

- Audio Conferencing: Audio conferencing is similar to telephonic communication, but it requires one-to-many good quality communication links. This service is suitable for delays $<150 \mathrm{~ms}$, jitter $<400 \mathrm{~ms}$, and bandwidth $>80 \mathrm{kbps}$. Hence, this service is also supported by TETRA.

\subsection{CONCLUSIONS AND FUTURE WORKS}

This study investigated video quality over TEDS using H.264/AVC and MPEG-4 video codecs. It proposed TENS framework to simulate uplink channel communication to analyse TEDS network performance for video communication. The results showed that the type of a dropped video frame, intermittent network connections, limited network bandwidth, and variety of video application severely affect the video quality. It is also observed that TEDS can only support limited frame rate video sequences which can be adopted to investigate the on-filed victims using Gait analysis. Furthermore, H.264/AVC and MPEG-4 are compared, then it is concluded that H.264/AVC offers better video quality 
due to its low bandwidth requirements and dropped-packet handling features. In future, we aim to enhance this work by including H.264/SVC video codec in our study.

\subsection{ACKNOWLEDGMENT}

The authors of this paper would like to thank the reviewers for their efforts to improve the quality of this work. This work is sponsored by Malaysian Ministry of Education under the High Impact Research Grant of University Malaya UM.C/625/1/HIR/ MOE/FCSIT/03.

\section{REFERENCES}

[1] S.Z. Hussain, Performance evaluation of H. 264/AVC encoded video over TETRA enhanced data service (TEDS), 2009.

[2] D.I. Axiotis, A.K. Salkintzis, and D. Xenikos, "IP Transmission over TETRA Packet Data Service: Simulation and Measurement Results", Wireless personal communications, Vol. 47 No. 4, December 2008, pp. 523-540.

[3] P. Stavroulakis, Terrestrial Trunked Radio: TETRA, Springer, 2007.

[4] S. Sohail and F. Somenzi, "Safety first: a two-stage algorithm for the synthesis of reactive systems", International Journal on Software Tools for Technology Transfer, Vol. 15 No. 5-6, October 2013, pp. 433-454.

[5] Y.C. Chang, M. Salim, and T.F. Tang, "Performance evaluation of MPEG-4 visual error resilient tools over a mobile channel", IEEE Transactions on Consumer Electronics, Vol. 49 No. 1, June 2003, pp. 6-13.

[6] D.I. Axiotis and D.G. Xenikos, "On the performance of TETRA short data service-transport layer", Wireless personal communications, Vol. 43 No. 4, December 2007, pp. 1121-1135.

[7] Y.C. Chang and M. Salim Beg, "MPEG-4 video error resilient tools performance evaluation and assessment", in Communications, Computers and signal Processing, 2003. PACRIM, 2003 IEEE Pacific Rim Conference on IEEE, 2003, pp. 70-72.

[8] A. Norouzi, M.S.M. Rahim, A. Altameem, T. Saba, A.E. Rad, A. Rehman, and M. Uddin, "Medical image segmentation methods, algorithms, and applications", IETE Technical Review, Vol. 31 No.3, June 2014, pp. 199-213.

[9] J.D.L. Delgado and J.M.R. Santiago, "Key Performance Indicators for QOS Assessment in TETRA Networks", International Journal of Mobile Network Communications \& Telematics (IJMNCT), Vol. 3 No. 6, December 2013, pp. 1-18.

[10] R.W. Ahmad, A. Gani, S.H.A. Hamid, F. Xia, and M. Shiraz, "A Review on mobile application energy profiling: Taxonomy, state-of-the-art, and open research issues", Journal of Network and Computer Applications, Vol. 58, December 2015, pp. 42-59.

[11] D.I. Axiotis and A.K. Salkintzis, "Impact of voice traffic on the performance of packet data transmission in TETRA networks", IEEE Transactions on Vehicular Technology, Vol. 57 No. 5, September 2008, pp. 3040-3052.

[12] T. Wiegand, G.J. Sullivan, G. Bjontegaard, and A. Luthra, "Overview of the H. 264/AVC video coding standard", IEEE Transactions on Circuits and Systems for Video Technology, Vol. 13 No. 7, July 2003, pp. 560-576.

[13] W. Li, "Overview of fine granularity scalability in MPEG-4 video standard", IEEE Transactions on Circuits and Systems for Video Technology, Vol. 11 No. 3, March 2001, pp. 301-317.

[14] G. Kandus, A. Hrovat, I. Ozimek, M. Smolnikar, and T. Ban-Zlatev, "Telemetry over TETRA Network", in 
Proceedings of the 2007 WSEAS Int. Conference on Circuits, Systems, Signal and Telecommunications, 2007, pp. 30-34.

[15] B. Dolanc and M. Judez, "Professional Mobile system-TETRA over IP and IP over TETRA", in EUROCON 2003. Computer as a Tool. The IEEE Region 8, IEEE, 2003, pp. 173-177.

[16] K.R. Carter and V. Jervis, Safety First: Reinvesting the Digital Dividend in Safeguarding Citizens, https://ssrn.com/abstract=1088706, 2008.

[17] J. Zhong, P. Mege, and C. Molko, "Research and simulation of HARQ protocol in TEDS wireless communication system", in 2011 IEEE 3rd International Conference on Communication Software and Networks (ICCSN), 2011, pp. $272-275$.

[18] F. Azizzadeh, S.A. Ghorashi, and A.M. Khodaian, "Performance evaluation and enhancement of random access procedure in TETRA networks", in 2010 6th Conference on Wireless Advanced (WiAD), 2010, pp. 1-5.

[19] S. Michail, A.P. Avramova, and L. Dittmann, "MIH based mobility for TETRA-LTE network", in Proceedings of ICT Innovations, 2013, pp. 180-189.

[20] S. AlQahtani, "Adaptive Resource Allocation Scheme for TETRA Networks with Multi-operators", in ACCESS 2011, The Second International Conference on Access Networks, 2011, pp. 1-6.

[21] F. Azizzadeh and S.A. Ghorashi, "Channel Allocation in TETRA Networks Using Channel Reservation", Australian Journal of Basic \& Applied Sciences, Vol. 5 No. 5, 2011.

[22] R.W. Ahmad, A. Gani, S.H.A. Hamid, M. Shiraz, A. Yousafzai, and F. Xia, "A survey on virtual machine migration and server consolidation frameworks for cloud data centers", Journal of Network and Computer Applications, Vol. 52, June 2015, pp.11-25.

[23] J. Liu, S. Hou, and H. Li, "Rate Control Algorithm of H. 264 on IPP for TETRA", in 3rd International Conference on Computer Science and Service System, 2014, pp. 250-253.

[24] J. Shuja, A. Gani, K. Bilal, A.U.R. Khan, S.A. Madani, S.U. Khan, and A.Y. Zomaya, "A Survey of Mobile Device Virtualization: Taxonomy and State of the Art", ACM Computing Surveys (CSUR), Vol. 49 No. 1, July 2016.

[25] L. Thornton, M. Chakraborty, and J. Soraghan, "Video over TETRA", in IEE Seminar on Tetra Market and Technology Developments (Ref. No. 2000/007), 2000.

[26] M. Salleh and J. Soraghan, "A Multisacle based Multistage Lattice Vector Quantization Video Coding for Robust Transmission over Wireless Channels", in 2007 IEEE International Conference on Signal Processing and Communications, 2007, pp. 388-391.

[27] S. BakariC, M. Borzic, D. BratkoviC, and V. Grga, "TETRA (terrestrial trunked radio)-technical features and application of professional communication technologies in mobile digital radio networks for special purpose services", in 47th International Symposium, ELMAR, 2005, pp. 307-310.

[28] H. Tang and Y. Zhang, "An dynamic channel allocation algorithm in TD-SCDMA trunking communication system", Journal of Chongqing University of Posts and Telecommunications (Natural Science Edition), 2012.

[29] J. Shuja, A. Gani, S. Shamshirband, R.W. Ahmad, and K. Bilal, "Sustainable Cloud Data Centers: A survey of enabling techniques and technologies", Renewable and Sustainable Energy Reviews, Vol. 62, 2016, 195-214. 
[30] S.A. Madani, K. Hayat, and S.U. Khan, "Clustering-based power-controlled routing for mobile wireless sensor networks", International journal of communication systems, Vol. 25 No. 4, 2012, pp. 529-542.

[31] M. Shiraz, A. Gani, R.W. Ahmad, S.A.A. Shah, A. Karim, Z.A. Rahman, "A lightweight distributed framework for computational offloading in mobile cloud computing", PloS one, Vol. 9 No. 8, 2014, e102270.

[32] P.G.V. Naranjo, M. Shojafar, H. Mostafaei, Z. Pooranian, and E. Baccarelli, "P-SEP: a prolong stable election routing algorithm for energy-limited heterogeneous fog-supported wireless sensor networks", The Journal of Supercomputing, Vol. 73 No. 3, 2016, pp. 733-755.

[33] M. Shojafar, S. Abolfazli, H. Mostafaei, and M. Singhal, "Improving channel assignment in multi-radio wireless mesh networks with learning automata", Wireless Personal Communications, Vol. 82 No. 1, 2015, pp. 61-80. 